\title{
EARLY RESULTS FROM NOAA-20 (JPSS-1) VIIRS ON-ORBIT CALIBRATION AND CHARACTERIZATION
}

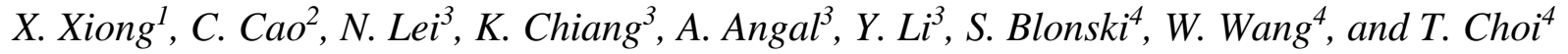 \\ ${ }^{1}$ Sciences and Exploration Directorate, NASA/GSFC, Greenbelt, MD 20771, USA \\ ${ }^{2}$ Center for Satellite Applications and Research, NOAA NESDIS, College Park, MD 20740, USA \\ ${ }^{3}$ Science Systems and Applications, Inc., 10210 Greenbelt Road, Lanham, MD 20706, USA \\ ${ }^{4}$ Earth Resources Technology, Inc., 5830 University Research Ct., College Park, MD 20740, USA
}

\begin{abstract}
Since launch in November 2018, the VIIRS on-board the NOAA-20 (or JPSS-1) satellite has completed its initial intensive on-orbit check-outs and several key calibration and validation activities scheduled to help evaluate sensor at launch performance. This paper provides a brief overview of NOAA-20 VIIRS on-orbit operation and calibration activities, presents early results derived from its on-board calibrators and lunar observations, and discusses potential improvements and future effort to assure sensor data product quality.
\end{abstract}

Index Terms - NOAA-20, JPSS-1, VIIRS, calibration

\section{INTRODUCTION}

The VIIRS is a key instrument on-board the NOAA-20 (N-20) satellite launched on November 18, 2017. Like its predecessor aboard the S-NPP launched in October 2011, the VIIRS collects data in 22 spectral bands. In addition to a day and night band (DNB), spectral bands (M1-M11 and I1-I3) with wavelengths from 0.41 to 2.2 $\mu \mathrm{m}$ are referred to as the reflective solar bands (RSB) and other bands (M12-16 and I4-I5) covering wavelengths from 3.7 to $12 \mu \mathrm{m}$ are referred to as the thermal emissive bands (TEB). M1-M5, M7, and M13 can make observations at either high- or low-gain and the DNB is capable of collecting data at three different gain stages.

The VIIRS sensor data records (SDR) provide key input for producing a range of environment data records (EDR) that support numerous applications for users from the operational and research community. As expected, the SDR quality strongly relies on instrument performance and its on-orbit calibration accuracy. Since launch, the N-20 VIIRS has conducted a series of onorbit check-outs and post-launch testing (PLT) to help characterize its at-launch performance. As a result of intensive calibration and validation efforts made at different stages of the PLT by different groups, including NOAA and NASA calibration teams, the N20 VIIRS SDR reached the beta maturity on January 25 , 2018 and then the provisional maturity status on February 19, 2018. The goal is to achieve validated maturity by end of May 2018. Following a brief description of N-20 VIIRS on-orbit operation and calibration, this paper presents early results derived from its on-board calibrators (OBCs) as well as lunar observations and discusses potential improvements and future effort that are critical for continuously producing high quality SDR and EDR.

\section{ON-ORBIT CALIBRATION}

As shown in Figure 1, the VIIRS on-orbit calibration is performed by the OBCs that include a solar diffuser (SD) and a solar diffuser stability monitor (SDSM) for the RSB and a blackbody (BB) for the TEB. Lunar observations are also made regularly as a supplement to instrument on-board calibration. The SD calibration is normally performed every orbit. The SDSM is currently operated on a daily basis. The BB warm-up/cool-down (WUCD), initially planned on a quarterly basis, will be executed less frequently in future operation. Like SNPP, lunar observations will be scheduled 8-9 times per year at nearly identical phase angles $\left(-51^{\circ}\right)$ [1-3]. The first lunar calibration for $\mathrm{N}-20$ VIIRS was performed on December 29, 2017 via a spacecraft roll maneuver. An 
example of lunar images from I1 and I2 spectral bands (middle 4 scans) is presented in Figure 2.

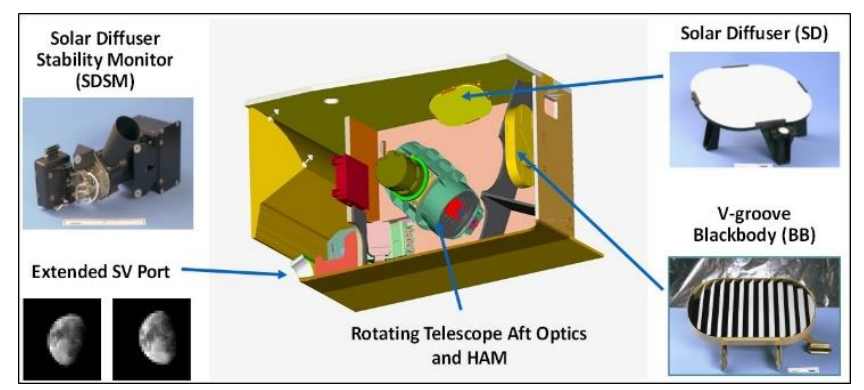

Fig. 1 VIIRS instrument and its on-board calibrators (OBCs).
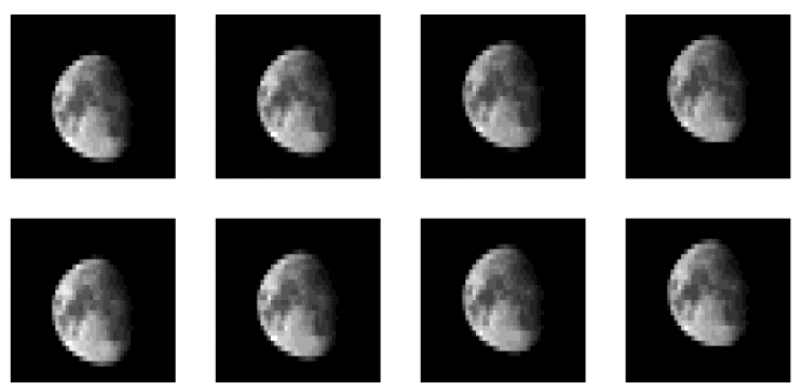

Fig. 2 Lunar impages (middle four scans) of N-20 VIIRS I1 (upper) and I2 (lower) from calibration event on December 29, 2017

Listed in the following are key events and calibration activities (starting dates) during N-20 VIIRS PLT:

- Nov 28, 2017: instrument turn on

- Nov 30, 2017: first SDSM operation

- Dec 08, 2017: first electronics self-test (Ecal)

- Dec 13, 2017: nadir aperture door (NAD) open

- Dec 18, 2018: first DNB calibration (VROP)

- Dec 29, 2017: first lunar calibration (roll maneuver)

- Jan 03, 2018: cryoradiator door open

- Jan 10, 2018: first BB WUCD

- Jan 25, 2018: calibration yaw maneuvers

- Jan 31, 2018: calibration pitch maneuver

- Feb 21, 2018: rotating telescope stow (3 days)

- Mar 12, 2018: mid-mission outgassing.

In addition to roll maneuvers, data collected from yaw maneuvers has already been applied to characterize SD and SDSM screen transmission as an effort to improve RSB calibration. Pitch maneuver data has been used to validate the TEB response versus scan-angle (RVS) and estimate the DNB high gain offsets. The VIIRS calibration maneuvers are designed and executed based on experience from MODIS [4]. Several special activities were performed from mid-February to midMarch to help investigate and mitigate the effect due to ice build-up that led to larger degradation of the LWIR spectral band responses.

\section{RESULTS AND DISCUSSION}

The RSB calibration is performed using the on-board SD panel placed behind a fixed solar attenuation screen (SAS) and a quadratic algorithm (cubic for SWIR). Each detector's responses to the sunlight reflected off the SD are used to derive a scaling factor (F-factor), which is inversely proportional to the detector's gain. Illustrated in Figure 3 are the measured F-factors using SD calibration data for the VIS/NIR spectral bands. Results are averaged over all detectors and for the HAM (half-angle mirror) side A. For the dual gain bands, only the high gain $(\mathrm{HG})$ results are presented here.

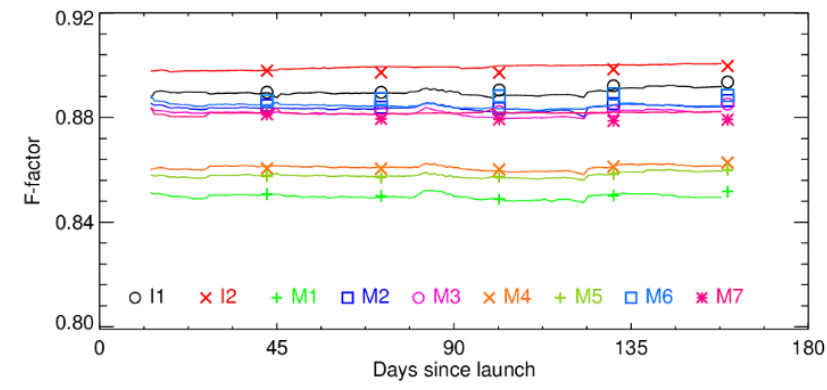

Fig. 3 F-factors of N-20 VIIRS VIS/NIR spectral bands deived from SD (solid curves) and lunar observations (symbols).

For comparison purposes, the F-factors derived from lunar observations are also presented in Figure 3 using symbols of the same colors for the corresponding spectral bands. On-orbit lunar calibration is very similar to the SD calibration except it uses the ROLO lunar model as its reference. The lunar F-factors shown in Figure 3 are normalized with their first points to SD Ffactors. As of May 1, 2018, the N-20 VIIRS has implemented 5 lunar calibration events. Results from both SD and lunar observations will be used together to track RSB calibration stability over entire mission. Additionally, lunar observations can be used to track on-orbit changes in sensor band-to-band registrations (BBR) [5]. To date, the N-20 BBR has been very stable.

In general, the RSB F-factors have been very stable, showing no sign of large degradation, such as that observed in S-NPP due to the telescope mirror coating contamination [6]. This is a significant performance 
enhancement, attributed to special effort made by the instrument vendor with lessons from S-NPP VIIRS testing and characterization. The F-factors used in the L1B or SDR data production include the corrections for the SD degradation, which is tracked by the on-board SDSM using 8 filtered detectors that cover the wavelengths from 0.41 to $0.93 \mu \mathrm{m}$. The results of SD on-orbit degradation are shown in in Figure 4.

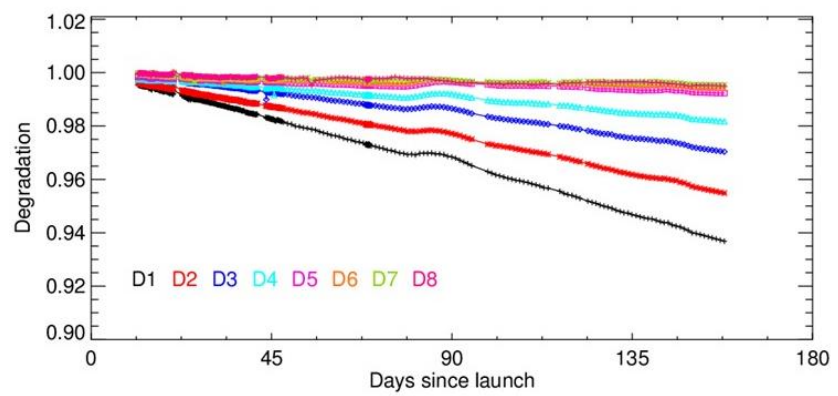

Fig. 4 N-20 VIIRS SD on-oribt degradation (D1-D8 wavelengths: $0.41-0.93 \mu \mathrm{m}$ )

The RSB signal-to-noise ratios (SNR) are calculated using detector responses over a range of signal levels during SD calibration that include the data collected when SD is partially illuminated. The measured SNR at different signals (radiances) are fitted to a function so that the SNR at typical radiance level can be derived. Listed in Table 1 are the current SNRs for the VIS/NIR spectral bands, including the ratios of measured SNR to specified SNR. Clearly, on-orbit SNR are much higher than the sensor design requirements. The SWIR bands have similar SNR performance. Thus far, the RSB SNR performance has been very stable.

Table 1 SNR (measured, Jan 2, 2018) for VIS and NIR spectral bands (I1-I2, M1-M7, HG only). Ratio = SNR (measured) / SNR (specified).

\begin{tabular}{|c|c|c|c|c|c|c|c|c|c|}
\hline Band & 11 & 12 & M1 & M2 & M3 & M4 & M5 & M6 & M7 \\
\hline SNR & 228 & 287 & 649 & 577 & 707 & 562 & 396 & 427 & 544 \\
\hline Ratio & 1.91 & 1.91 & 1.84 & 1.52 & 1.70 & 1.55 & 1.64 & 2.14 & 2.53 \\
\hline
\end{tabular}

The TEB are located on the cold focal plane assemblies (CFPA) with temperature controlled by a cryoradiator. The CFPA temperatures reached the nominal operating temperature a few days after opening the cryoradiator door on Jan 3, 2018. The first BB WUCD was carried out on Jan 10, 2018, providing an opportunity for a comprehensive characterization of the TEB. Except for a few cases, such as BB WUCD or safe hold events, the BB has been nominally controlled at $292.5 \mathrm{~K}$. Its short- term stability and spatial uniformity are demonstrated in Figure 5 by the scan-by-scan temperatures averaged over its 6 thermistors and their standard derivations. As expected, the $\mathrm{BB}$ performance in the nighttime orbits is more stable and uniform than in the daytime orbits.
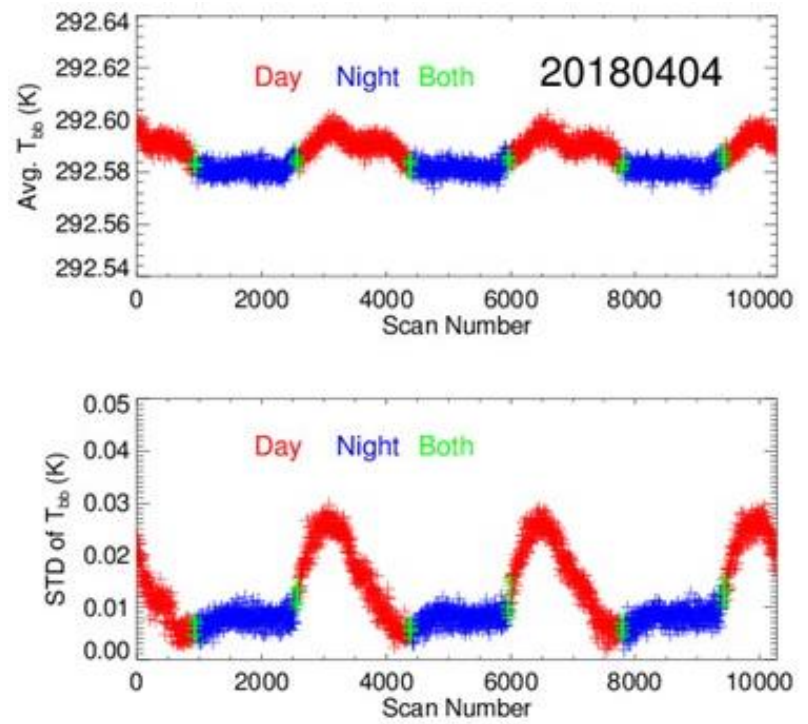

Fig. 5 N-20 VIIRS BB scan-by-scan temperatures averaged over its 6 thermistors (upper plot) and their corresponding standard derivations (lower plot). The nightime/daytime data ara shown in blue/red color and data with both daytime and nighttime are plotted in green color.

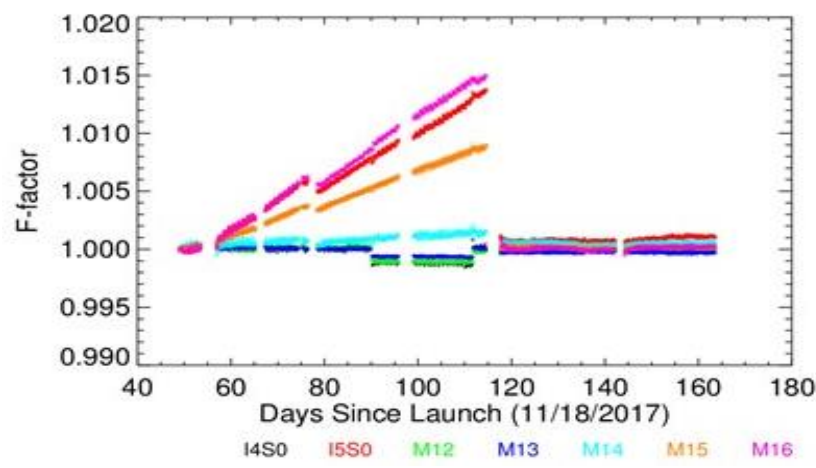

Fig. 6 N-20 VIIRS TEB F-factors (band averaged, HAM side $\mathrm{A})$.

Shortly after all the TEB became operational, it was found that some LWIR bands (M15, M16, and I5) had a slow degradation that was not seen in the S-NPP. Following a series of diagnostic activities, the root cause of this apparent degradation was determined to be ice deposition on the windows and/or the Integrated Filter Assembly (IFA) within the LWIR dewar. After a mid-mission outgassing operation on March 12, 2018, the LWIR bands regained their responses (gains) to 
their expected levels. Shown in Figure 6 are the TEB Ffactors (1/gains) to date. Apart from LWIR degradation, the F-factor trends have several noticeable data gaps and discontinuities that are primarily due to the changes of instrument operating conditions. Currently, all TEB performance parameters are as expected based on prelaunch calibration and meet nearly all the specified requirements. Results shown in Table 2 are the TEB noise equivalent temperature difference (NEdT) at typical temperatures that are derived using data from BB WUCD. The ratios of the measured to specified NEdT are also provided. Ratio values of less than 1 indicate better performance (smaller detector noise).

Table 2 N-20 TEB NEdT derived from BB WUCD (April 9, 2018) for spectral bands (I4-I5, M12-M16, HG only). Ratio $=$ NEdT $($ measured $) /$ NEdT $($ specified $)$.

\begin{tabular}{|c|c|c|c|c|c|c|c|}
\hline Band & 14 & 15 & $\mathrm{M} 12$ & $\mathrm{M} 13$ & $\mathrm{M} 14$ & $\mathrm{M} 15$ & $\mathrm{M} 16$ \\
\hline NEdT & 0.383 & 0.338 & 0.100 & 0.037 & 0.047 & 0.023 & 0.031 \\
\hline Ratio & 0.15 & 0.23 & 0.25 & 0.35 & 0.51 & 0.33 & 0.43 \\
\hline
\end{tabular}

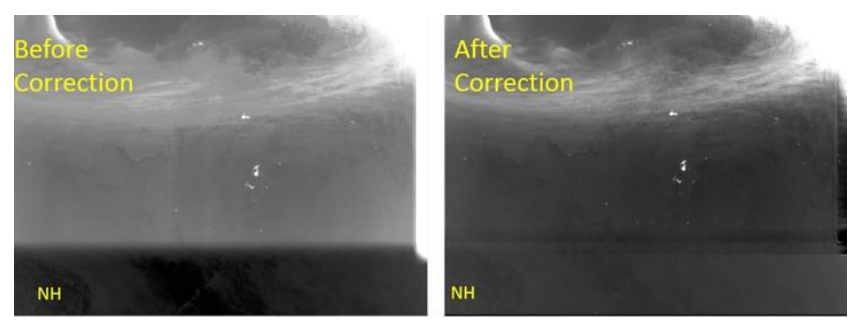

Fig. 7 N-20 VIIRS DNB images before and after a straylight correction (see text for details).

Like S-NPP, N-20 VIIRS DNB also shows noticeable straylight contamination, especially in the extended zone. Figure 7 shows a pair of DNB images in Alaska region (March 17, $2018 \sim 13: 00$ UTC), one before and one after a straylight correction (performed by the NOAA STAR VIIRS SDR Team). In general, the straylight correction performs well. Residual effects in the extended zone and straylight boundary regions, however, still exist and require further improvements.

\section{IMPROVEMENTS AND FUTURE WORK}

Since launch, a number of calibration improvements have been made. Using data collected during spacecraft yaw maneuvers, the SD and SDSM screen transmission functions are derived, updated and used for on-orbit calibration. Compared to pre-launch measurements, onorbit yaw maneuver data provide fine details, both geometrically (more angles) and spectrally (more wavelengths). The TEB RVS characterized using pitch maneuver data shows good agreement with pre-launch results. DNB straylight correction strategy is based on lessons from S-NPP and needs extra effort to address effects in the extended zone (not in S-NPP). Similar to S-NPP, the lunar long-term trend will be used to support RSB solar calibration. From the perspective of longterm data records, the calibration consistency between S-NPP and N-20 VIIRS will likely be a major challenge and requires more studies.

\section{ACKNOWLEDGEMENTS}

We would like to acknowledge dedicated efforts and contributions made by other members of the VIIRS calibration teams at NASA and NOAA and support from the mission operation team (MOT).

\section{REFERENCES}

[1] Xiong X., J. Butler, K. Chiang, B. Efremova, J. Fulbright, N. Lei, J. McIntire, H. Oudrari, J. Sun, Z. Wang, and A. Wu, "VIIRS On-orbit Calibration Methodology and Performance," JGR Vol. 119, Issue 9, pp 5065-5078, 2014

[2] Cao, C., X. Xiong, F. Deluccia, R. Wolfe, and F. Weng, "Early On-Orbit Performance of the Visible Infrared Imaging Radiometer Suite Onboard the Suomi National PolarOrbiting Partnership (S-NPP) Satellite," IEEE TGRS, vol.52, no.2, pp.1142-1156, 2014

[3] Xiong, X., J. Sun, J. Fulbright, Z. Wang, and J. Butler, "Lunar Calibration and Performance for S-NPP VIIRS Reflective Solar Bands,” IEEE TGRS, Vol 54, Issue 2, pp 1052-1061, 2016

[4] Butler, J., X. Xiong, B. Barnes, F. Patt, J. Sun, and K. Chiang, "An overview of Suomi NPP VIIRS calibration maneuvers", Proc. SPIE 8510, 85101J, 2012

[5] Wang, Z., X. Xiong, and Y. Li, "Update of VIIRS onorbit spatial parameters characterized with the Moon," IEEE Transactions on Geoscience and Remote Sensing, vol. 53, issue 10, pp. 5486-5494, 2015

[6] De Luccia, F., D. Moyer, E. Johnson, K. Rausch, N. Lei, K. Chiang, X. Xiong, J. Fulbright, E. Haas, G. Iona, "Discovery and characterization of on-orbit degradation of the Visible Infrared Imaging Radiometer Suite (VIIRS) Rotating Telescope Assembly (RTA)," Proc. SPIE, 8510, 85101A, 2012 